Recepción: 19/ 06 / 2018

Aceptación: 21/ 08 / 2018

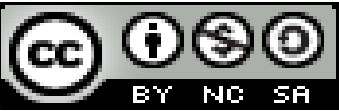

Ciencias de la educación

Publicación: 05/ 10 / 2018

Artículo de investigación

\title{
La Educación Superior. Rendimientos académicos. Factores asociados
}

Higher education. Academic returns Associated factors

Ensino Superior Retornos acadêmicos Fatores associados

\author{
Nancy M. Cedeño-Macías I \\ nancy.cedeño@hotmail.com \\ María F. Pin-Navarro II \\ mariapin.navarro@hotmail.com
}

Correspondencia: nancy.cedeño@ hotmail.com

\footnotetext{
${ }^{\text {I }}$ Magister en Gerencia Educativa, Licenciada en Ciencias de la Educación, Especialidad Media, Escuela Fiscal Jaime Rodos Aguilera, Santa, Ecuador, Docente de Nivelación de la Universidad Técnica de Manabí, Portoviejo, Ecuador.

${ }^{\text {II }}$ Magister en Gerencia Educativa, Licenciada en Ciencias de la Educación, Mención Psicología y Orientación Vocacional, Unidad Educativa Fiscal del Milenio Olmedo, Portoviejo, Ecuador; Docente de Nivelación de la Universidad Técnica de Manabí, Portoviejo, Ecuador.
} 


\section{Resumen}

Este trabajo presenta una exploración exhaustiva en las literaturas referente al tema, de estas averiguaciones hace alusión a la Educación Superior, rendimientos académicos y los posibles factores asociados en estudiantes universitarios. La comprensión de los factores que principalmente incurren en el rendimiento académico en estudiantes universitarios permitiría al menos, entre otros, predecir resultados académicos y poder hacer un estudio sobre su acaecimiento en la calidad educativa que se espera y ser una herramienta para la toma de decisiones en esta materia.

Palabras clave: rendimiento académico, factores asociados, estudiantes universitarios, educación superior.

\section{Abstract}

This work presents an exhaustive exploration in the literature referring to the subject, of these findings refers to Higher Education, academic performance and the possible associated factors in university students. Understanding the factors that mainly affect the academic performance of university students would at least, among others, predict academic results and be able to make a study about their occurrence in the educational quality that is expected and be a tool for decision making in this matter.

Keywords: academic performance, associated factors, university students, higher education.

\section{Resumo}

Este trabalho apresenta uma exploração exaustiva na literatura referente ao tema, destes achados refere-se ao Ensino Superior, desempenho acadêmico e os possíveis fatores associados em estudantes universitários. Compreender os fatores que mais afetam o desempenho acadêmico dos universitários poderia, ao menos, entre outros, predizer resultados acadêmicos e ser capaz de fazer um estudo sobre sua ocorrência na qualidade educacional esperada e ser uma ferramenta para tomada de decisão em este assunto. 
Palavras chave: desempenho acadêmico, fatores associados, estudantes universitários, ensino superior.

\section{Introducción}

Ternemos la intención de conceptualizar la educación superior como los estudios posteriores a la educación diversificada, donde el estudiantado escoge un área a fin en la cual desee continuar sus estudios con la aspiración de laborar en ese campo. La educación universitaria prepara a las personas para enfrentarse al mercado laboral, las especializa y capacita para el desempeño de sus quehaceres. (Cruz Sancho S, Sandí Delgado J. 2014)

En el orbe la Educación Superior tiende a ajustarse a los cambios que ha sufrido el capitalismo en todos los órdenes: social, político, económico, ideológico, cultural, los objetivos medulares universitarios son: la docencia, la investigación y la difusión de la cultura. La misión de estos centros es preparar, técnicos, profesionistas y científicos que estudien y aporten al desarrollo de las ciencias y las humanidades y que contribuyan con propuestas para la solución incluyente de los problemas de la sociedad mundial y de la nación en que se encuentren. Los egresados se ubican en las diversas ocupaciones que brinda el sistema, pero es necesario procurar que salgan con conciencia social de los retos para el desarrollo de sus actividades; aunque no todos asumen esa responsabilidad, una vez, egresados sino los valores del sistema que requiere fuerza de trabajo que sirva al proceso de acumulación de la riqueza en pocas manos. (Calderón Ortiz, G, Zamora Fonseca, R, \& Medina Ruíz, G. 2017).

En ese mismo sentido parte de los estudiantes cuando ingresan a los centros de enseñanza superior ya tienen asumidos los valores del sistema por lo que su preparación tiene el objetivo de lograr ascender rápidamente en la escala social y obtener oportunidades, altos salarios, poder, etcétera, sea en empresas capitalistas o en el sector gubernamental. Los valores éticos o son desconocidos por ellos o bien los ven, la mayoría, como una carga que no merece tenerse en cuenta. Así hay una gama de conductas que se asumen y que lleva a algunos en su formación prepararse para beneficio propio y egoísta, así como para servir a los poderes fácticos o institucionales sean de organizaciones capitalistas o gubernamentales. (Calderón Ortiz, G, Zamora Fonseca, R, \& Medina Ruíz, G. 2017). 
La masificación de la matrícula, la adquisición de habilidades y destrezas operacionales, computacionales y estratégicos que se desarrollan caracterizan las nuevas tendencias de la educación superior, al margen de la institución paradigmático-histórica. Estas habilidades y destrezas se desarrollan en el contexto de la sociedad de la información, del aprendizaje y del conocimiento. (Características de la Educación Superior del Siglo XXI. 2011)

Hecha la observación anterior, al irrumpir en la Educación Universitaria, debemos recordar que vivimos en una nueva sociedad, "la sociedad del conocimiento" (Kenza, 2011); no obstante la Universidad es una institución que tiene la posibilidad de adaptarse a los cambios que plantea la sociedad de conocimiento, que siempre se verá afectada por el creciente intercambio comercial y cultural, especialmente entre países diferentes, es claro que esto obliga a que cada participante del mercado de la globalización sea cada vez más eficaz y eficiente, de tal manera que esté capacitado y con grandes oportunidades de competir con los más altos niveles de productividad y calidad

En literatura estudiada hace referencia que el futuro de la universidades está ligado estrechamente al desenvolvimiento y desarrollo de capacidades para promover, incentivar y sobre todo consolidar cambios conceptuales capaces de satisfacer las urgentes demandas de la sociedad, especialmente de los sectores menos favorecidos, preparando personas con un alto espíritu de superación y colaboración, con la predisposición de adaptarse adecuadamente para responder de manera eficiente a un mundo que se transforma de modo precipitado e imprevisto, cuyas implicaciones sociales y culturales se ven reflejadas en una realidad que nos enseña que es necesario darle un "valor agregado" a la utilización del conocimiento para su posterior incorporación a la producción y el trabajo, lo cual si se lo lleva de la manera correcta y adecuada, colabora en gran medida para la prosperidad y el bienestar de una nación, siempre con la mentalidad de servir principalmente a los más necesitados.(Pinos Vélez E . 2013).

En el espacio investigativo especialistas, asistente a La Declaración de la Conferencia Mundial sobre la Educación Superior, promulgaron que Citamos (...)

Si se carece de instituciones de educación superior e investigación adecuadas que formen una masa crítica de personas calificadas, ningún país podrá garantizar un auténtico desarrollo 
endógeno y sostenible, los países en desarrollo y los países pobres no podrán acortar la distancia que los separa de los países desarrollados industrializados (UNESCO, 1998).

Es de un inmenso valor para todos aquellos que están dedicados en el tema, conocer que el papel de la Universidad es muy importante en el desarrollo social, político y económico de las naciones, es conocido que las tendencias mundiales para los centros de educación superior deban reorientar sus objetivos y dejar atrás la visión que impide una plena y activa participación en el desarrollo, promover una proyección social. (Pinos Vélez E. 2013)

De esta manera la misión y visión de las Universidades debe ir más allá del de proveer e inculcar a sus educandos conocimientos básicos y académicos que posteriormente puedan ser aplicados, no siempre la Universidad lo enseña todo, se debe mejorar especialmente en el área de la investigación, pero una investigación científica orientada a mejorar las condiciones de vida de los sectores menos privilegiados de la sociedad, es decir, ser consciente de las necesidades de la gente y de cómo mejorar utilizando recursos propios..(Pinos Vélez E . 2013)

Entendemos pertinentemente detenernos en expresar que la atención a la capacitación de profesores de educación superior también debe ser un tema estratégico que tiene mucha relación con el compromiso de educar mejor a quienes tienen el privilegio de ingresar a una institución de Educación Superior. El primordial propósito de la educación es el ser humano más no la asignatura, es por eso que se debe fortalecer la personalidad del educando y del educador, por consiguiente, debe haber necesariamente una capacidad educadora y educable, en donde la interacción de los dos elementos fundamentales, uno con la capacidad de influenciar y otro con la capacidad de ser influenciado (Fernández - Sarramona, 1984). No solo se aprenden conocimientos y habilidades, sino también valores y sentimientos que siempre serán expresados en la conducta del ser humano, basados en el establecimiento de relaciones afectivas fundamentadas en el respeto mutuo, la comprensión y la aceptación.

En los momentos actuales la universidad debe asimilar la idea de que las principales fuentes de productividad y competitividad en la economía global dependen principalmente de la creatividad de la generación de conocimientos y un correcto procesamiento de la información, la misma que debe estar encaminada a favorecer a los sectores más vulnerables, las propuestas planteadas en la 
actualidad no están diseñadas o no cuentan con planes estratégicos fortalecidos, más aún cuando en la actualidad, en el Ecuador, existen nuevas normativas que las Instituciones deben acoger y cumplir, sin embargo, aún no se establece lineamientos como por ejemplo, para una inclusión educativa..(Pinos Vélez E . 2013)

Según Pinos Vélez E (2013) el desafío, de las Instituciones de Educación Superior y de manera especial de las Universidades es el de afrontar el reto de enfrentar un mundo en el cual los sistemas productivos están en constante transformación, pero siempre desde la propia realidad en la que vive un país, con propuestas encaminadas al fortalecimiento de los valores fundamentales que se pueda tener como Universidad para con la Sociedad en donde la Educación a cualquier nivel, deje de ser excluyente.

\section{Antecedentes: contexto de las universidades públicas latinoamericanas}

Desde la década de los años 90 en el nuevo contexto mundial, la tesis que prevalece es que la educación superior estatal no se desarrollará como debe, si depende exclusivamente de los fondos públicos, razón por la cual se han impulsado políticas y acciones tendientes a la venta de servicios para adquirir recursos propios, no sin problemas y contradicciones respecto de su función. Esta realidad es particularmente importante en América Latina, ya que la educación superior pública históricamente ha desempeñado un papel vital en la promoción de la democracia. Sus intelectuales han enriquecido el pensamiento nacional. Muchas veces las universidades han servido como vínculos decisivos en la modernización política, así como en la promoción que han realizado de la legitimidad y estabilidad política. Ha cumplido, en buena medida, un papel motor en la movilidad social y desempeña un papel fundamental en la trasmisión de la cultura nacional y en el desarrollo de la identidad nacional.

Según datos del Banco Interamericano de Desarrollo (BID) (1996), la educación superior en América Latina y el Caribe es una actividad prioritaria y no marginal, debido a la propia modernización e integración de la región en una economía globalizada, que exige un cuadro de profesionales altamente competente. Por ello, desempeña un papel decisivo, porque es condición para el progreso económico. Este desarrollo requiere de una reorientación y de recursos, que el BID está anuente a pro mover y, de hecho, fue el máximo donante externo de recursos en las 
décadas de 1960 y 1970. Solo entre 1962, que fue el año de su primera inversión, y 1984 desembolsó para estos propósitos \$540.732.000. A criterio de personeros del BID, (1996) las universidades poseen cuatro funciones básicas en la región: liderazgo académico, formación para las profesiones, formación técnica y perfeccionamiento, y educación superior general. Por ello, requieren de un adecuado diagnóstico y abordaje. (Garbanzo Vargas G 2012)

Rivero, H. J. (1999), esboza que en América Latina y el Caribe se espera que la educación superior sea conducida de manera más eficaz, en relación con el incremento continuo de la inversión en capital humano, efectuada en los sistemas educativos privados y públicos, en vista de que está aumentando el papel desempeñado por esta región en la economía mundial. La educación es un medio, herramienta y estrategia esencial para ayudar a los pueblos a prepararse ante los embates de la globalización y para enfrentar sus posibles repercusiones (Rivero, 1999).

América Latina continúa realizando esfuerzos en busca de un mejor equilibrio entre los graduados y el mercado laboral, lo que evidencia que el acceso a la educación superior en igualdad de condiciones debe ir acompañado de un proyecto de desarrollo económico, que reduzca las brechas de desempleo en la región, es decir con responsabilidad social. (Garbanzo Vargas G 2012.

\section{Rendimiento académico en la Educación Superior. Factores asociados}

Se conceptualiza que el rendimiento académico es una medida de las capacidades del alumno, que expresa lo que éste ha aprendido a lo largo del proceso formativo, además de suponer la capacidad del alumno para responder a los estímulos educativos. (Martínez Villafuerte J P, Salazar Poveda R. 2013)

Existen problemas comunes en todos los países de nuestro entorno cultural y económico como: el bajo rendimiento académico, el excesivo tiempo invertido en el estudio de una titulación, el abandono de los estudios. (Martínez Villafuerte J P, Salazar Poveda R. 2013)

Es merecido señalar que el rendimiento académico del estudiantado universitario constituye un factor imprescindible en el abordaje del tema de la calidad de la educación superior, debido a que es un indicador que permite una aproximación a la realidad educativa. (Díaz, Peio, Arias, 
Escudero, Rodríguez, Vidal, 2002). No se puede hablar de calidad de la educación superior sin conocer a fondo indicadores asociados al rendimiento académico de los estudiantes universitarios, pues este análisis representa un monitoreo estratégico en cuanto al desempeño académico y por ende la utilización de los recursos que el Estado invierte. (Garbanzo Vargas, G M 2007),

Es importante expresar que las culturas del rendimiento académico en la Educación Superior parecen ser en la coyuntura mundial actual aún más valiosos, debido al dinamismo que experimenta el sector universitario en el marco de una sociedad caracterizada por el rápido avance del conocimiento, la fluidez en la transmisión de la información y los cambios acelerados en las estructuras sociales. En ese contexto adquiere valor la calificación del capital humano y ello va en estrecha vinculación con los resultados e investigaciones sobre el rendimiento académico de los estudiantes universitarios. (Garbanzo Vargas, G M 2007),

Es oportuno destacar que, desde un punto de vista práctico, lo frecuente es identificar rendimiento con resultados, debiendo distinguirse entre éstos dos categorías: inmediatos y diferidos. Los primeros estarían determinados por las calificaciones que obtienen los alumnos durante su carrera hasta la obtención del título correspondiente y se definen en términos de éxito/fracaso en relación a un determinado período temporal. Por otro lado, el rendimiento diferido hace referencia a su conexión con el mundo del trabajo, en términos de eficacia y productividad, se vincula, sobre todo, con criterios de calidad de la institución. (Tejedor F.J, Valcárcel Muñoz Repiso A.G 2007)

Se evidencia que el rendimiento académico, por ser multicausal, encierra una monumental capacidad explicativa de los distintos factores y espacios temporales que intervienen en el proceso de aprendizaje. Existen diferentes aspectos que se asocian al rendimiento académico, entre los que intervienen componentes tanto internos como externos al individuo. (Garbanzo Vargas, G M 2007),

Son muchos los motivos que pueden llevar a un alumno a mostrar un pobre rendimiento académico, muchas están directamente relacionadas al factor psicológico, como la poca motivación, el desinterés o las distracciones en clase, que dificultan la comprensión de los 
conocimientos desarrollados por el docente y el estudiante y termina afectando al rendimiento académico a la hora de las evaluaciones. (Armenta N.G, Pacheco C y Pineda E 2008)

En este sentido, si bien el rendimiento académico está vinculado a la aptitud existen además otros factores que afectan en el rendimiento académico como son factores relacionados con el estudiante (sexo, genero, estado civil, nivel socioeconómico, carrera universitaria, horario de clases, alimentación, realización de ejercicio, tiempo dedicado al ocio), factores psicológicos (problemas psicoafectivos, uso de cualquier tipo de drogas), factores relacionados con el entorno familiar, factores demográficos, factores pedagógicos lo que se relaciona con la calidad de enseñanza actual vs la calidad de enseñanza escolar, el número de alumnos por maestro, los métodos y materiales didácticos utilizados, la motivación de los estudiantes y el tiempo dedicado por los profesores a la preparación de sus clases, y factores regionales .(Martínez Villafuerte J P, Salazar Poveda R . 2013)

Por otra parte, Garbanzo plantea que son múltiples los factores que han de intervenir sobre el éxito académico, pudiendo agruparlos en determinantes propios del estudiante, determinantes sociales y determinantes institucionales. A continuación, siguiendo el camino directo del tema se ofrecen los principales factores asociados al rendimiento académico:

Competencia cognitiva, Motivación, Condiciones cognitivas, Auto concepto académico, Autoeficacia percibida, Bienestar psicológico, Satisfacción y abandono con respecto a los estudios, asistencia a clases, Inteligencia, Aptitudes, Sexo, Formación académica previa a la Universidad y Nota de acceso a la universidad. (Martínez Villafuerte J P, Salazar Poveda R . 2013)

Se reseña que la motivación es un pilar fundamental del proceso enseñanza- aprendizaje, tanto así que Chomsky plantea: "La verdad del asunto es que aproximadamente el 99 por ciento de la enseñanza es lograr que los alumnos se interesen por la materia"

En este orden de ideas se puede citar que la competencia cognitiva se define como la autoevaluación de la propia capacidad del individuo para cumplir una determinada tarea cognitiva, su percepción sobre su capacidad y habilidades intelectuales. Está relacionada con la influencia ejercida en el entorno familiar e incide en distintas variables que se asocian con el 
éxito académico tales como: la persistencia, el deseo del éxito, expectativas académicas del individuo y la motivación. El afecto de los padres hacia el estudiante se asocia con el establecimiento de una alta competencia académica percibida y con la motivación hacia el cumplimiento académico. (Pelegrina, García y Casanova, 2002).

Necesario destacar que el auto concepto como factor asociados al rendimiento académico está fuertemente vinculado con la motivación del estudiante y sus resultados académicos. Se puntualiza como el conjunto de percepciones y creencias que una persona posee sobre sí misma, es así como la mayoría de variables personales que orientan la motivación parten de las creencias y percepciones que el individuo tiene sobre aspectos cognitivos; la capacidad percibida por parte del estudiante, el rendimiento académico previo y creer que la inteligencia se desarrolla a partir del esfuerzo académico, contribuyen a mejorar un auto concepto académico positivo..( Garbanzo Vargas, G M 2007), la satisfacción personal tiene un papel importante en el desempeño académico, así mismo las condiciones cognitivas son estrategias de aprendizajes que el estudiante lleva a cabo relacionadas con la selección, organización, y elaboración de los diferentes aprendizajes. Se definen como condiciones cognitivas del aprendizaje significativo. La orientación motivacional da pie a la adopción de metas, que determinan en gran medida las estrategias de aprendizaje que el estudiante emplea y repercuten en su rendimiento académico. La percepción que el estudiante construya sobre factores como la evaluación, el tipo de materia, la complejidad de la materia y el estilo de enseñanza, influyen en las estrategias de aprendizaje. El uso de mapas conceptuales, hábitos de estudio, horas asignadas estudio, y las prácticas académicas son algunas estrategias de aprendizaje utilizadas por los estudiantes. (Garbanzo Vargas, G M 2007),

El Bienestar psicológico, da cuenta de un estado de equilibrio y ausencia de patologías emocionales/psicológicas, más allá de los cuadros considerados normales de neurosis, emerge como factor de base en el rendimiento.

Referente a la Asistencia a clases tiene impacto en la motivación y que la ausencia se relaciona con problemas de repetición y abandono a los estudios. (Vázquez C 2012) 
La Complejidad en los estudios, es una variable que siempre muestra relaciones importantes con el rendimiento académico al igual que las condiciones de las aulas, servicios, plan de estudios y formación del profesorado. De relevancia similar se citan los Servicios institucionales de apoyo, que la institución ofrece a los estudiantes, como son sistemas de becas, servicio de préstamos de libros, asistencia médica, apoyo psicológico, pedagógico, entre otros. (Vázquez C 2012).

Relacionado con las variables Sociales como los estudios de los padres población de residencia pueden tener incidencia sobre la regularidad académica de los alumnos es prácticamente nula al igual que la situación laboral de los padres. (Tejedor Tejedor, 2003)

Se tiene referente que según resultados del estudio por Martínez Villafuerte J (2013) en su investigación determinó que los factores determinantes que se encuentran relacionados con el desempeño académico fueron: número de horas clase día, número de horas de estudio por día, horas de sueño diarias, número de horas de ocio diarias.

Estudios llevados a cabo por Vélez Van, Roa (2005), con estudiantes universitarios en Colombia; Carrión (2002) en Cuba; Valle, González, Núñez, Martínez, Pineñor, (1999) en la Universidad de Coruña, en España y Montero, Villalobos, en la Universidad de Costa Rica, se ha abordado el tema del rendimiento académico a partir de variables cognitivas, emocionales y socioeconómicas, entre las que se incluyen resultados de la educación secundaria, pruebas del Estado, el examen de ingreso a la universidad, las pruebas de aptitudes intelectuales, factores psico- sociales (consumo de alcohol y otro tipo de sustancias), rasgos de personalidad y factores relacionados con el estado emocional hacia el estudio, calificaciones obtenidas, composición familiar, el interés vocacional, los hábitos de estudio y el nivel académico de los padres..( Garbanzo Vargas, G M 2007),

En otras series realizada en la Universidad de Asunción. Paraguay se ha notificado que factores asociados al rendimiento escolar que responden a las características socio culturales y económicas. (Suárez Enciso S, Elías R, Zarza D. 2016).

\section{Consideraciones finales}

La comprensión de los factores que principalmente incurren en el rendimiento académico en estudiantes universitarios permitiría al menos, entre otros, predecir resultados académicos y poder 
hacer un estudio sobre su acaecimiento en la calidad educativa que se espera y ser una herramienta para la toma de decisiones en esta materia.

\section{Referencias Bibliográficas}

Armenta N.G, Pacheco C y Pineda E. (2008). Factores socioeconómicos que intervienen en el desempeño académico de los estudiantes Universitarios de la Facultad de Ciencias Humanas de La Universidad Autónoma de Baja California, México; 11 (1): 153 -165. Recuperado de: http://revistasinvestigacion.unmsm.edu.pe/index.php/psico/article/viewFile/3888/311.

Cruz Sancho S, Sandí Delgado J. (2014). Importancia de la educación superior en el Desarrollo Profesional Para La población Estudiantil. Recuperado de. http://sedici.unlp.edu.ar/bitstream/handle/10915/46200/Documento_completo.pdf?sequence=3

Calderón Ortiz, Gilberto, Zamora Fonseca, Raquel, \& Medina Ruíz, Geidy. (2017). LA EDUCACIÓN SUPERIOR EN EL CONTEXTO DE LA GLOBALIZACIÓN. Revista

Universidad y Sociedad, 9(3), 310-319. Recuperado de http://scielo.sld.cu/scielo.php?script=sci_arttext\&pid=S2218-

$36202017000300048 \& \operatorname{lng}=\mathrm{es} \& \operatorname{tn} \mathrm{ln}=\mathrm{es}$

Características de la Educación Superior del Siglo XXI. (2011). Recuperado de https://consultoraepisteme.wordpress.com/2011/01/15/caracteristicas-de-la-educacion-superiordel-siglo-xxi/

Carrión Pérez, E. (2007). Validación de características al ingreso como predictores del rendimiento académico en la carrera de medicina. Revista Cubana de Educación Media Superior, 1(16), ¿Recuperado de http://scielo.sld.cu/scielo.php? script=sci_arttext\&pid=S0864-214 $2002000100001 \& \operatorname{lng}=\mathrm{es} \& \mathrm{nrm}=\mathrm{i}$

Díaz, M., Peio, A., Arias, J., Escudero, T., Rodríguez, S., Vidal, G. J. (2002). Evaluación del Rendimiento Académico en la Enseñanza Superior. Comparación de resultados entre alumnos procedentes de la LOGSE y del COU. En: Revista de Investigación Educativa, 2(20), 357-383. 
Fernández A. y Sarramona J. (1984). La Educación, Constantes y problemática Actual. Barcelona: CEAC. 12 ed.

Garbanzo Vargas G (2012). Educación Superior pública en América Latina: Características y Desafíos, Revista GUAL, Florianópolis, 5 (1), pp. 216- 227. Recuperado de https://periodicos.ufsc.br/index.php/gual/article/viewFile/1983-4535.2012v5n1p216/21943

Garbanzo Vargas, G, Factores asociados al rendimiento académico en estudiantes universitarios, una reflexión desde la calidad de la educación superior pública. (2007). Revista Educación, 31. Recuperado de en:<http://www.redalyc.org/articulo.oa?id=44031103> ISSN 0379-7082

Kenza El Gahli (2011). Los Retos de la Universidad Marroquí en la Sociedad del Conocimiento. Facultad de Letras y Ciencias Humanas. Universidad Hassan II - Mohammedia. Magriberia $\mathrm{N}^{\circ} 4$. Recuperado de: dialnet.unirioja.es/descarga/ articulo/3897581.pd La Educabilidad Recuperado de: http://webs.uvigo.es/jtarrio/OBRA\%2 LITERARIA/LA\%20EDUCABILIDAD.pdf

Martínez Villafuerte J P, Salazar Poveda R. (2013). Factores determinantes sobre el rendimiento académico En estudiantes de las facultades de medicina, enfermería, Microbiología, psicología, arquitectura y derecho de la Universidad Católica del ecuador. Recuperado de http://repositorio.puce.edu.ec/bitstream/handle/22000/7373/11.27.001757.pdf;sequence=4

Pelegrina, Saniago García, Linares M. C, Casanova, Pedro F. (2002). Parentingstyles and adolescents' academic perfomance En: Infancia y aprendizaje. Revista electrónica USAL), 25 (2), 147-168.

Pinos Vélez E. (2013). La educación universitaria: exigencias y desafíos. alteridad. Revista de Educación ISSN No. 1390-325X $8 \quad$ (1), 97-105. Recuperado dehttps://dspace.ups.edu.ec/bitstream/123456789/8326/1/La\%20educaci\%C3\%B3n\%20universit aria\%20exisgencias\%20y\%20desaf\%C3\%ADos.pdf

Rivero, H. J. (1999). “Equidad en la educación.” Revista Iberoamericana de Educación. 23. Madrid. Recuperado de http://www.campus.oei.org/revista/rie23a03.htm. 
Suárez Enciso S, Elías R, Zarza D . (2016). Factores Asociados al Rendimiento Académico de Estudiantes de Paraguay: Un Análisis de los Resultados del TERCE. Revista Iberoamericana sobre Calidad, Eficacia y Cambio en Educación, 14(4), 11 3-133 doi:10.15366/reice2016.14.4.006.

Tejedor F.J, Valcárcel Muñoz Repiso A.G. (2007) Causas del bajo rendimiento del estudiante universitario (en opinión de los profesores y alumnos). Propuestas de mejora en el marco del EEES. Revista de Educación; (342): 443- 473. Recuperado de http://www.revistaeducacion.mec.es/re342/re342_21.pdf

UNESCO (1998). Proyecto Principal de Educación en América Latina y el Caribe Boletín 40. Santiago, Chile: agosto. UNESCO. Recuperado de: http://unesdoc.unesco.org/images/0010/001047/104761s.pdf

Vélez Van, M. A., Roa, N. C. (2005). Factors as sociat ed with academic perfomance in medical students. En: PSIC. Educación Médica. 2 (8), 1-10.

Valle Arias, A.; González Cabanach, R.; Núñez Pérez, J.; Martínez Rodríguez, S; Pineñor Aguin, I. (1999). Un modelo causal sobre los determinantes cognitivo-motivacionales del rendimiento académico. Revista de Psicología General Aplicada. 52 (4), 499-519

Vázquez C, Cavallo M A, Aparicio S, Muñoz B, Robson C, Ruiz L. Secreto M, Sepliarsky P, Escoba M E. (2012). Factores de impacto en el rendimiento académico universitario. Un estudio a partir de las percepciones de los estudiantes. Recuperado de https://www.fcecon.unr.edu.ar/webnueva/sites/default/files/u16/Decimocuartas/vazquez_c_factor es_de_impacto_en_el_rendimiento_academico.pdf 